\title{
Neurorestorative process, law, and mechanisms
}

\author{
This article was published in the following Dove Press journal: \\ Journal of Neurorestoratology \\ 2 February 2015 \\ Number of times this article has been viewed
}

\author{
Hongyun Huang ${ }^{1-3}$ \\ Lin Chen $^{3-5}$ \\ 'General Hospital of Armed Police \\ Forces, ${ }^{2}$ Beijing Rehabilitation Hospital \\ of Capital Medical University, ${ }^{3}$ Beijing \\ Hongtianji Neuroscience Academy, \\ ${ }^{4}$ Tsinghua University Yuquan Hospital, \\ ${ }^{5}$ Medical Center, Tsinghua University, \\ Beijing, People's Republic of China
}

\begin{abstract}
Neurorestoration, or restoration of neurological function, can be achieved by different strategies through a series of neurorestorative mechanisms, including neuroprotection, neuromodulation, neuroplasticity, immunomodulation, axonal regeneration and sprouting, remyelination or neurorepair, neuroregeneration or neurogenesis, and neuroreplacement. Unfortunately, these mechanisms have been studied and viewed in isolation. The aim of this review is to generalize all attacking insults and restoring mechanisms as a complete neurorestorative process, then analyze the changing process of dynamic interaction between neural insults and restorative mechanisms, and highlight neurorestorative law, ie, "smaller or larger self-restoration always occurs by nature while facing insults, active and appropriate medical intervention is able to better restore functions and/or structures". This review also briefly describes several important neurorestorative mechanisms.
\end{abstract}

Keywords: neurorestoration, strategies, mechanisms, neurorestorative process, neurorestorative law, neurorestoratology

\section{Introduction}

Neurorestoration is a process to restore, promote, or maintain the integrity of neurological functions by neurorestorative strategies, including physical (electronic or magnetic), chemical (medicine or factors), biological (cell therapy, molecule, bioengineering, and tissue engineering), surgical, or other kind of intervention, which usually bring anatomic structural and/or functional restoration simultaneously. ${ }^{1}$ Neurorestorative mechanisms, as the basis of central nervous system (CNS) neurorestorable theory, include neuroprotection, neuromodulation, neuroplasticity (neurosynapsis, neural circuit, or network reconstruction), immunomodulation, axonal regeneration and sprouting, remyelination or neurorepair, neuroregeneration or neurogenesis, neuroreplacement, and angiogenesis. ${ }^{2-4}$ What is the interaction between neural insults and restorative mechanisms? What is the relationship between the different neurorestorative mechanisms? Herein we explain their inner relationship and briefly describe several important neurorestorative mechanisms, including neuromodulation, neuroprotection, neuroplasticity, and neurogenesis or neuroregeneration in the CNS.

\section{Neurorestorative process and the law}

Regardless of the various causes, the neural insults most likely to make neurons (their soma and fibers) and glia or environment initiate pathophysiological processes may be larger and severer or smaller and milder, that is, the neurons involved may 
be partly in an unhealthy state, or undergo excitotoxicity, abnormal autophagy, or death (necrosis or apoptosis). Thus, restoring mechanisms by nature or interventional strategies must react to those insults through some or all neurorestorative mechanisms. Unfortunately, the mechanisms for the different insults have always been studied and viewed in isolation, but should be investigated as a whole because of their close inner relationship. When the attacking and restoring mechanisms are generalized as a complete neurorestorative process (Figure 1A), the changing process of dynamic interaction between neural insults and restorative mechanisms is clearly demonstrated. The neurorestorative process can be viewed as a physiological state, with three pathophysiological stages, ie, early, middle, and final. It illustrates the full dynamic neural course of all animal life, including that of human beings. When there is an insult, there must be restorative action in both the central and peripheral nervous systems.

Self-restoration, however, may not be strong enough in the pathophysiological stages, so appropriate intervention is necessary to enhance functional restoration. More importantly, from this neurorestorative process, we have found that the neurorestorative law is, "smaller or larger self-restoration always occurs by nature while facing insults, active and appropriate medical intervention is able to better restore functions and/or structures". We believe that all neural activity in the physiological and pathophysiological states follows this law in terms of physical, psychological, and spiritual functioning. However, more exploration needs to be done in the future with regard to the extent, mechanisms, and strategies of neurorestoration.

\section{Physiological state}

When neural degeneration or damage is mild, physiological functions are maintained mainly via self-restoring mechanisms, including neuroprotection, neuroplasticity, neuromodulation, and/or immunomodulation.

\section{Pathophysiological states}

When neurons cannot maintain their functional and/or structural physiological integrity in response to attacking insults via self-restoring mechanisms, they will continue to become worse in pathophysiological state.

\section{Early stage}

Living unhealthy neurons without function, but with integral structure, result from past or ongoing insults. Prevention of

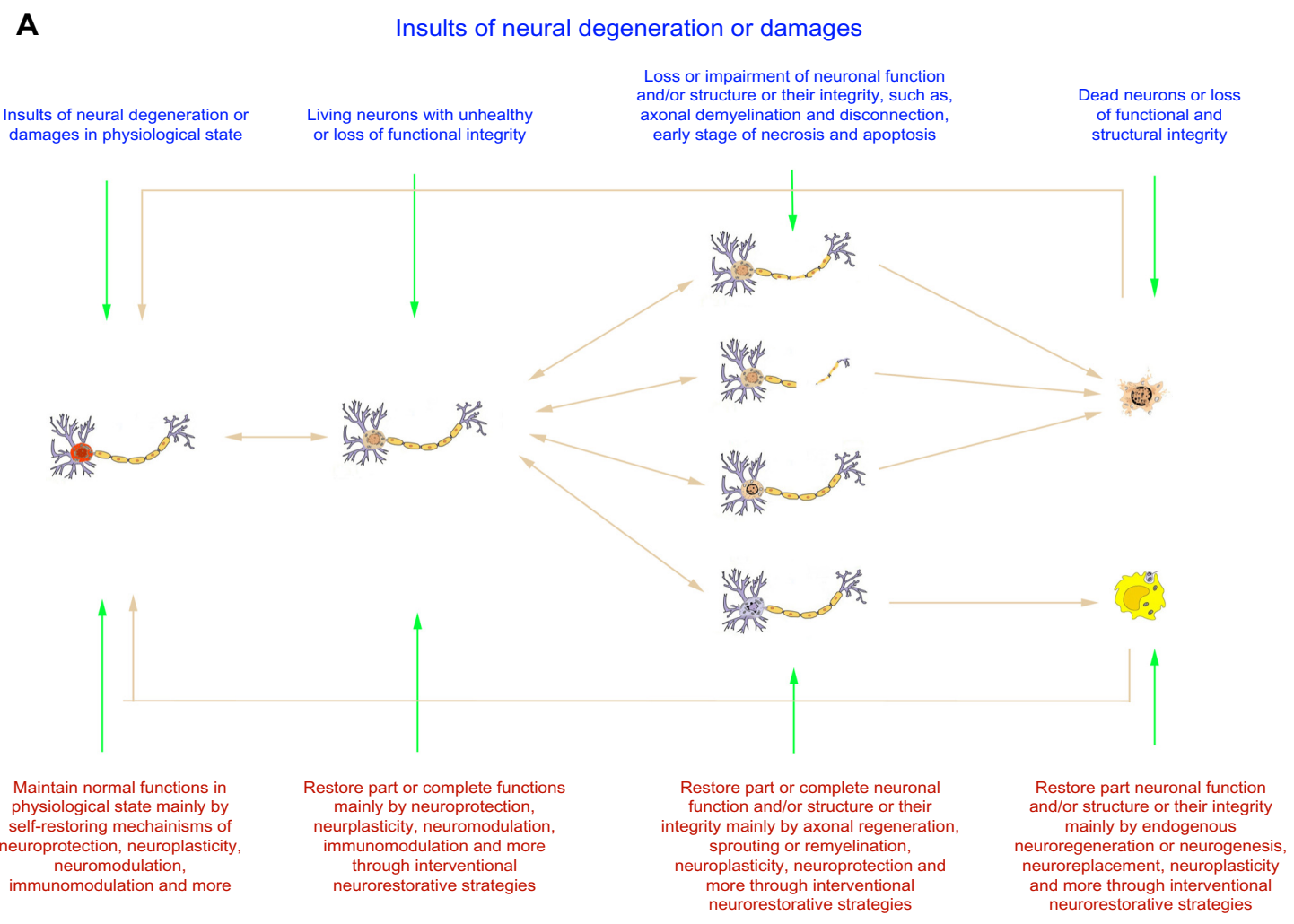

Neurorestorative mechanisms

Figure I (Continued) 
B

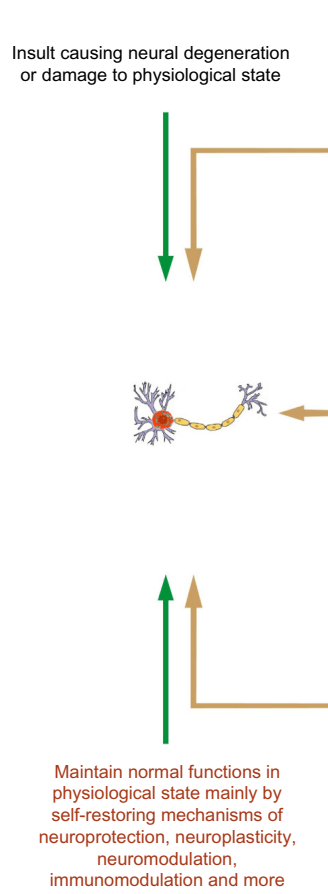

C

\section{。}

Insult causing neural degeneration or damage to physiological state
Insult causing neural degeneration or damage Loss or impairment of neuronal function
and/or structure or their integrity, such as, Living neurons that are unhealthy axonal demyelination and disconnection, or have lost functional integrity early stage of necrosis and apoptosis
Dead neurons or loss of functional and

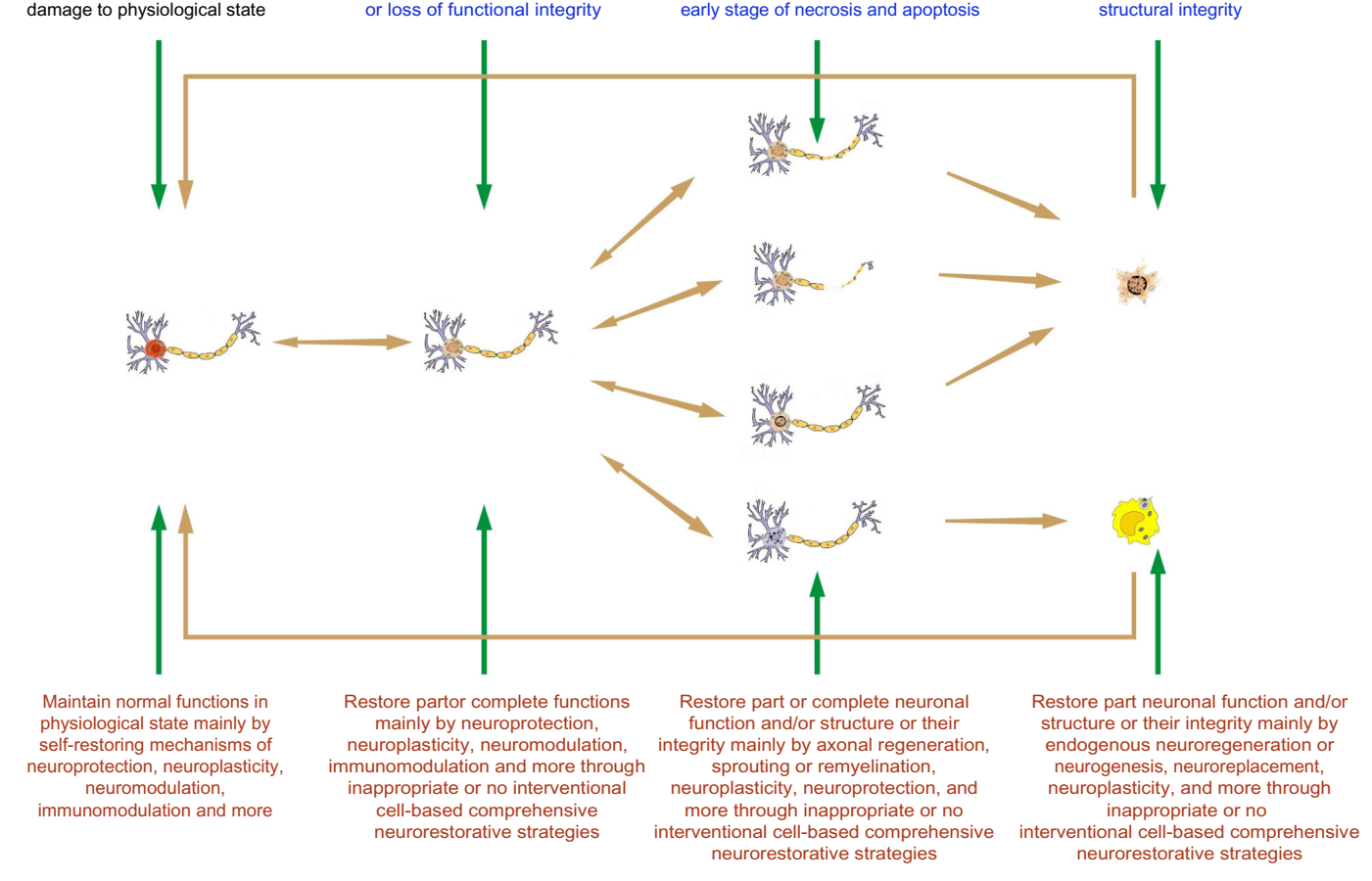

Neurorestorative mechanisms

Figure I (A) Neurorestorative process, (B) neurorestorative process with effective interventional strategies, and (C) neurorestorative process with inappropriate interventional strategies.

Notes: (A) The direction of arrows keeps in balance, which means neurological functions and/or structures remain stable with self-restoring or interventional strategies. (B) The arrows become bigger from right to left, which means neurological functions and/or structure become better with effective interventional strategies. (C) The arrows become bigger from left to right, which means neurological functions and/or structures become worse with inappropriate interventional strategies. 
further deterioration and restoration of a part or complete function can be achieved by neuroprotection, neuroplasticity, neuromodulation, and immunomodulation.

\section{Middle stage}

Loss or impairment of neuronal function and/or structural integrity by past insults can be completely or partly reversed by axonal regeneration, sprouting, remyelination, neurorepair, and neuroplasticity.

\section{Final stage}

Dead neurons and loss of functional or structural integrity caused by past insults that do not exist anymore can be restored by endogenous neuroregeneration or neurogenesis, neuroplasticity, and neuroreplacement.

The entire process of the neuron lifespan from normal state to death (Figure 1A) is probably an example of the entire nervous system, and how the different restoring mechanisms play their roles in the whole process. Clearly, neurorestoration is the only term that can accurately reflect the implications of the neurorestorative process. Each important neurorestorative mechanism has some role in the physiological or pathophysiological stages, but not in the whole process.

Unfortunately, this dynamic process of interaction between neural insults and restorative mechanisms, especially in the CNS, has been ignored for over a century in the community. Why? First, the conventional viewpoint does not acknowledge the possibility of CNS restoration. Second, there has been no overall perspective in this field. Third, the mechanisms for the different types of impairment have been studied and viewed in isolation. Fourth, the majority of neuroscience researchers have focused on neuroregeneration and overlooked other mechanisms, and this continues to be the case.

Figure 1A shows that it is clear that no single neurorestorative mechanism can exert all roles in the whole neurorestorative process. For example, neuroregeneration and neurogenesis may only play a role in the final stage of neuron death. But how much neuroregeneration or neurogenesis can occur? Even if it really happens, it does not mean function is restored. So, overemphasizing neuroregeneration is possibly a century directional mistake; we now believe that it should not be a main study direction in the field of neurorestoratology in the near future.

Figure 1A also contains other important information, as follows:

- In view of the process of developing disease, insults to single cells can generally be clearly seen at distinct stages, but insults to the entire nervous system appear to occur at a mixture of stages.

- In view of the mode of action of the different neurorestorative mechanisms, theoretically they can be separated, but in practice they work together and cannot be separated.

- Importantly, in CNS, currently neuroprotection, neuroplasticity, neuromodulation, and immunomodulation, but not neuroregeneration or neurogenesis, exert main roles in entire neurorestorative process.

- In view of the time and degree of neurorestorative treatment, we believe that the earlier, the better, and the earlier, likely the easier. In the early or middle pathophysiological stages, damaged cells have a chance to recover through appropriate interventional strategies. This implies that early interventional neurorestorative strategies should be recommended and even more, preventive interventional neurorestorative strategies or whatever stimulates endogenous neurorestorative mechanisms should be recommended.

- In view of the balance in the changing process of disease, if neurorestorative mechanisms with appropriate interventional strategies are stronger than insults, the balance of the entire neurorestorative process may return (Figure 1B), otherwise the result due to insults will become worse (Figure 1C).

- With regard to optimizing neurorestoration, one strategy may work through several mechanisms, but comprehensive strategies are likely to restore more functions, so combinations should be paid more attention.

For decades, people have expected rapid structural repair when the CNS is damaged, particularly the spinal cord. Although this expectation has not generally been met, there have been some cases of restoration of nerve function in patients with chronic spinal cord injury who received cell therapy after their natural neurological recovery had plateaued. ${ }^{4-7}$ In patients with complete chronic spinal cord injury, early functional recovery is mainly the result of signaling repair, neuromodulation, or unmasking by neurotrophins and a change in the local microenvironment as a result of cell therapy, instead of neuroregeneration or neurogenesis, which proven by fast functional recovery in many patients within only a few days of cell transplantation.

Under general conditions in the CNS, clinical neurorestorative mechanisms are very complex and comprehensive (Figures 1A and 2). One strategy may restore neurological function through several neurorestorative mechanisms, and different strategies may share the same mechanisms. Any type of neurorestoration may originate from one or all of 
the mechanisms listed above. However, the current research indicates that functional recovery comes mostly from neuromodulation, neuroprotection, and immunomodulation, often from neuroplasticity, axonal remyelination, or neurorepair, and sometimes from neurogenesis or neuroregeneration and angiogenesis. ${ }^{8,9}$ Neuroreplacement may be an important mechanism in Parkinson's disease, but not in other diseases or damage affecting the CNS.

\section{Neuromodulation}

Thirty years ago, neuromodulation was considered to be a physiological process of regulation of neuronal activity by neuromodulators or neurotransmitters, ${ }^{10}$ such as acetylcholine, norepinephrine, histamine, serotonin, dopamine, and a variety of neuropeptides, glutamate, D-serine, gamma-aminobutyric acid, and glycine. ${ }^{11-16}$ Drugs targeting neurotransmitters can affect the related neural systems.

Neuromodulation has also been considered to be a therapeutic method for restoring neurological function by neurostimulation. ${ }^{17}$ Neurostimulators include auditory brainstem implants, brain-computer interfaces, deep brain stimulation, functional electrical stimulation, repetitive transcranial magnetic stimulation, peripheral nerve stimulation, spinal cord stimulation, transcranial direct current stimulation, and intrathecal drug delivery systems. ${ }^{18}$

Until the last decade, neuromodulation was considered as a method that carries out interactions based on principles of nerve cell physiology, by neuromodulation techniques which include neurostimulation by implantable devices, ${ }^{19}$ and as a mechanism for neurological functional recovery by controlled electrical stimulation. ${ }^{20}$

Neuromodulation, defined by the International Neuromodulation Society as a therapeutic alteration of activity through electrical and drug stimulation, both are introduced by implanted devices and can be used to modulate neural function. ${ }^{21}$ More accurately speaking, neuromodulation should also include physical or interventional regulation of neural function through neuromodulators or neurotransmitters, and cell therapy ${ }^{5}$ implanted devices are not necessarily required.

Thus, neuromodulation is appropriately conceived of as the mechanisms and strategies used to activate neural function by implanted devices, neurotransmitters or neuromodulators, and cell therapy. As a neurorestorative mechanism, the term neuromodulation refers to modulation of function by using implantable neurostimulatory devices and medication or via

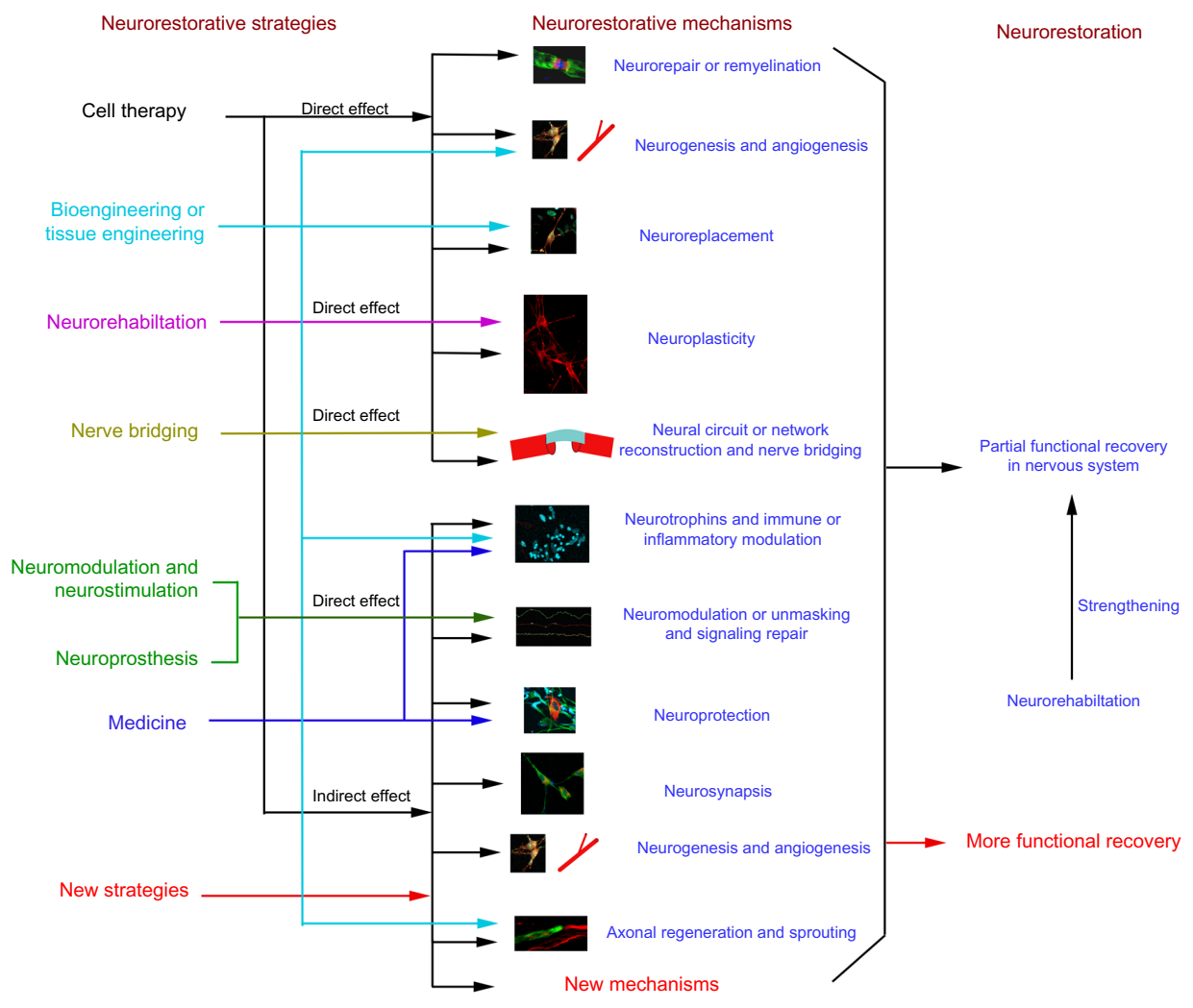

Figure 2 Neurorestorative schematic diagrams for implementation.

Notes: Copyright (C) 2013. Dove Medical Press. Reproduced from Huang H, Sharma HS. Neurorestoratology: one of the most promising new disciplines at the forefront of neuroscience and medicine. Journal of Neurorestoratology. 20। 3; 1:37-4I. ${ }^{52}$ 
modulation of ongoing neuronal spiking by the action of neurotransmitters or neuromodulators on local postsynaptic receptors. Further, implantable neurostimulatory devices and medication may also play a role by causing neuronal spiking and/or modulating ongoing neuronal spiking.

\section{Neuroprotection}

Neuroprotection refers to the mechanisms and strategies protecting against neuronal injury and degeneration in the nervous system, and includes all acute neural disorders and chronic neurodegenerative diseases.

As one of the neurorestorative mechanisms, neuroprotection works by limiting excitotoxicity and oxidative stress, and coping with inflammatory changes, iron accumulation, protein aggregation, and mitochondrial dysfunction. ${ }^{22-25}$

The goal of neuroprotection is to limit neuronal dysfunction/ death when damage or degeneration is occurring, and to maintain the integrity of neural function as far as possible. As one of the neurorestorative therapeutic strategies, neuroprotection includes free radical trappers/scavengers, anti-excitotoxic agents, apoptosis inhibitors, anti-inflammatory agents, neurotrophic factors, metal ion chelators, ion channel modulators, gene therapy, and cell therapy. ${ }^{26-28}$

\section{Neuroplasticity}

Neuroplasticity encompasses both synaptic plasticity and nonsynaptic plasticity, which changes in the neural pathways and synapses in response to changes in behavior and environmental/neural activity, as well as changes resulting from bodily injury. Neuroplasticity continues but declines with advancing age. ${ }^{29,30}$ It occurs at the cellular level due to learning or on a larger scale at the cortical level in response to injury and in healthy development, learning, memory, and recovery from neural damage.

William James proposed the idea that the brain and its functions were not fixed throughout adulthood in $1890,{ }^{31}$ but Ramón Y Cajal concluded in the early 20th century that CNS neurons could not regenerate. ${ }^{32}$ However, Hebb and Konorski introduced the idea of neural plasticity at a functional level, and Liu and Chambers observed sprouting while Raisman demonstrated sprouting based on experimental quantitative electron microscopic techniques. ${ }^{33-36}$

Throughout the 20th century, it was believed that the structure of the brain was relatively immutable after a critical period during early childhood. However, there is increasing evidence showing that many aspects of the brain remain plastic even in adulthood, and retain the ability to change throughout the lifespan. ${ }^{37}$ Neuroplasticity can be classified into two types. One is the physiological processes that are an obligatory response to neural development, learning, memory, sensory input, motor act, thinking, cognition association, awareness, and other neural activity. The other is the obligatory pathogenetic processes that occur in response to neural damage or degenerative insults. When an animal suffers from a disease or injury, nature intervenes and offers a mechanism to repair the structural damage and restore its function for survival.

\section{Neurogenesis and neuroregeneration}

Neurogenesis refers to the regeneration of neurons from neural stem cells and progenitor cells. The first evidence of adult mammalian neurogenesis in the cerebral cortex was demonstrated by Altman in $1962,{ }^{38}$ who subsequently discovered that adult neurogenesis occurred in the dentate gyrus of the hippocampus. ${ }^{39}$

Many factors have the ability to affect the rate of neurogenesis in the hippocampus. Cerebral ischemia, ${ }^{40}$ epileptic seizures, ${ }^{41}$ and bacterial meningitis ${ }^{42}$ may induce neurogenesis. On the other hand, chronic stress and aging can result in decreased neuronal proliferation. ${ }^{43-45}$ Exercise and an enriched environment can aid the survival of neurons and promote newborn cells to integrate into the hippocampus. ${ }^{46-49}$

The term neuroregeneration also includes generation of new neurons, glia, axons, myelin, and synapses, so is similar to the concept of neurogenesis, but broader. Generally, neurogeneration is taken to mean axonal regeneration. ${ }^{50}$ Neuroregeneration occurs to a significant degree in the peripheral nervous system. ${ }^{51}$ However, unlike in peripheral nervous system injury, injury to the CNS is not followed by extensive or robust regeneration, being limited by the inhibitory influences of the glia and extracellular environment.

In summary, it is now possible to promote functional neurorestoration via various strategies either in isolation or in combination, based on CNS neurorestorable theory. The concept of CNS neurorestorable theory, neurorestorative process, and law helps people to understand the real value of what they are doing and where they stand. Further, new perspectives on CNS neurorestorable theory, neurorestorative process, and neurorestorative law can help people clearly see current advancements in many viewpoints of neuroscience and clinical disciplines related to neurorestoration. The mechanisms of functional recovery are well known to vary at different pathophysiological stages (Figure 1A-C), but currently their activity cannot be well controlled or adjusted because we still do not clearly understand these mechanisms 
in detail. Definitions of neurorestorative mechanisms often overlap with each other and are not well delineated, it is not easy to differentiate them clearly in clinic. Given that the fixed pattern and route vary case by case, neurorestoration is a realistic route to reach the target, similar to the rule that fluid follows the path of least resistance in hydrodynamics. Excitingly, more research attention is now being paid to neurorestorative mechanisms at a higher level.

\section{Disclosure}

The authors report no conflicts of interest in this work.

\section{References}

1. International Association of Neurorestoratology. Beijing Declaration of International Association of Neurorestoratology (IANR). Cell Transplant. 2009;18:487.

2. Huang H, Chen L, Sanberg P. Cell therapy from bench to bedside translation in CNS neurorestoratology era. Cell Med. 2010;1:15-46.

3. Karussis D, Karageorgiou C, Vaknin-Dembinsky A, et al. Safety and immunological effects of mesenchymal stem cell transplantation in patients with multiple sclerosis and amyotrophic lateral sclerosis. Arch Neurol. 2010;67:1187-1194.

4. Lima C, Pratas-Vital J, Escada P, Hasse-Ferreira A, Capucho C, Peduzzi JD. Olfactory mucosa autografts in human spinal cord injury: a pilot clinical study. J Spinal Cord Med. 2006;29:191-203.

5. Huang H, Chen L, Wang H, et al. Influence of patients' age on functional recovery after transplantation of olfactory ensheathing cells into injured spinal cord. Chin Med J (Engl). 2003;116:1488-1491.

6. Huang $\mathrm{H}$, Wang $\mathrm{H}$, Chen $\mathrm{L}$, et al. [Influence factors for functional improvement after recovery after olfactory ensheathing cell for chronic spinal cord injury]. Zhongguo Xiu Fu Chong Jian Wai Ke Za Zhi. 2006;20:434-438. Chinese.

7. Guest J, Herrera LP, Qian T. Rapid recovery of segmental neurological function in a tetraplegic patient following transplantation of fetal olfactory bulb-derived cells. Spinal Cord. 2006;44:135-142.

8. Toft A, Scott DT, Barnett SC, Riddell JS. Electrophysiological evidence that olfactory cell transplants improve function after spinal cord injury. Brain. 2007;130 Pt 4:970-984.

9. Yamamoto M, Raisman G, Li D, Li Y. Transplanted olfactory mucosal cells restore paw reaching function without regeneration of severed corticospinal tract fibres across the lesion. Brain Res. 2009;1303:26-31.

10. Zimmerman EA, Liotta A, Krieger DT. beta-Lipotropin in brain: localization in hypothalamic neurons by immunoperoxidase technique Cell Tissue Res. 1978;186:393-398.

11. Linster C, Fontanini A. Functional neuromodulation of chemosensation in vertebrates. Curr Opin Neurobiol. 2014;29C:82-87.

12. Santos-Carvalho A, Álvaro AR, Martins J, Ambrósio AF, Cavadas C. Emerging novel roles of neuropeptide $\mathrm{Y}$ in the retina: from neuromodulation to neuroprotection. Prog Neurobiol. 2014;112:70-79.

13. Schmidt SL, Chew EY, Bennett DV, Hammad MA, Fröhlich F. Differential effects of cholinergic and noradrenergic neuromodulation on spontaneous cortical network dynamics. Neuropharmacology. 2013;72:259-273.

14. Bardawee SK, Alzweiri M, Ishaqat AA. D-serine in neurobiology: CNS neurotransmission and neuromodulation. Can J Neurol Sci. 2014;41:164-176.

15. Dacks AM, Reale V, Pi Y, et al. A characterization of the Manduca sexta serotonin receptors in the context of olfactory neuromodulation. PLoS One. 2013;8:e69422.

16. Turpin F, Dallérac G, Mothet JP. Electrophysiological analysis of the modulation of NMDA-receptors function by D-serine and glycine in the central nervous system. Methods Mol Biol. 2012;794:299-312.
17. Schröttner O. [Electric neuromodulation-a new concept in the treatment of chronic pain]. Wien Med Wochenschr. 1978;128(23):721-723. German.

18. International Neuromodulation Society. Neuromodulation: an emerging field. July 30, 2014. Available from: http://www.neuromodulation.com/ treatment. Accessed September 26, 2014.

19. Rise MT. Instrumentation for neuromodulation. Arch Med Res. 2000;31:237-247.

20. Andrews RJ. Neuroprotection trek-the next generation: neuromodulation I. Techniques - deep brain stimulation, vagus nerve stimulation, and transcranial magnetic stimulation. Ann N Y Acad Sci. 2003;993:1-13.

21. International Neuromodulation Society. About neuromodulation. July 30, 2014. Available from: http://www.neuromodulation.com/ about-neuromodulation. Accessed September 26, 2014.

22. Andersen JK. Oxidative stress in neurodegeneration: cause or consequence? Nat Med. 2004;10 Suppl:S18-S25.

23. Dunnett SB, Björklund A. Prospects for new restorative and neuroprotective treatments in Parkinson's disease. Nature. 1999; 399(Suppl 6738):A32-A39.

24. Hall R, Murdoch J. Brain protection: physiological and pharmacological considerations. Part II: The pharmacology of brain protection. Can J Anaesth. 1990;37:762-777.

25. Zádori D, Klivényi P, Szalárdy L, Fülöp F, Toldi J, Vécsei L. Mitochondrial disturbances, excitotoxicity, neuroinflammation and kynurenines: novel therapeutic strategies for neurodegenerative disorders. J Neurol Sci. 2012;322:187-191.

26. Lange KW, Youdim MB, Riederer P. Neurotoxicity and neuroprotection in Parkinson's disease. J Neural Transm Suppl. 1992;38:27-44.

27. LiY, Chen L, Zhao Y, et al. Intracranial transplant of olfactory ensheathing cells can protect both upper and lower motor neurons in amyotrophic lateral sclerosis. Cell Transplant. 2013;22 Suppl 1:S51-S65.

28. Ma Y, Liu W, Wang Y, et al. VEGF protects rat cortical neurons from mechanical trauma injury induced apoptosis via the MEK/ERK pathway. Brain Res Bull. 2011;86:441-446.

29. Pascual-Leone A, Freitas C, Oberman L, et al. Characterizing brain cortical plasticity and network dynamics across the age-span in health and disease with TMS-EEG and TMS-fMRI. Brain Topogr. 2011;24:302-315.

30. Pascual-Leone A, Amedi A, Fregni F, Merabet LB. The plastic human brain cortex. Ann Rev Neurosci. 2005;28:377-401.

31. James W. The Principles of Psychology. New York, NY, USA: Henry Holt; 1890.

32. Cajal SR. Degeneration and Regeneration of the Nervous System. Oxford, UK: Oxford University Press; 1928.

33. Raisman G. Neuronal plasticity in the septal nuclei of the adult rat. Brain Res. 1969;14:25-48.

34. Liu CN, Chambers WW. Intraspinal sprouting of dorsal root axons. AMA Arch Neurol Psychiatry. 1958;79:46-61.

35. Hebb DO, Penfield W. Human behaviour after extensive bilateral removal from the frontal lobes. Arch Neurol Psychiatry. 1940;44:421-436.

36. Konorski J. Conditioned Reflexes and Neuron Organization. London, UK: Cambridge University Press; 1948.

37. Rakic P. Neurogenesis in adult primate neocortex: an evaluation of the evidence. Nat Rev Neurosci. 2002;3:65-71.

38. Altman J. Are new neurons formed in the brains of adult mammals? Science. 1962;135:1127-1128.

39. Altman J. Autoradiographic investigation of cell proliferation in the brains of rats and cats. Anat Rec. 1963;145:573-591.

40. Jin K, Wang X, Xie L, et al. Evidence for stroke-induced neurogenesis in the human brain. Proc Natl Acad Sci U S A. 2006;103: 13198-131202.

41. Parent JM, Elliott RC, Pleasure SJ, Barbaro NM, Lowenstein DH. Aberrant seizure-induced neurogenesis in experimental temporal lobe epilepsy. Ann Neurol. 2006;59:81-91.

42. Gerber J, Tauber SC, Armbrecht I, Schmidt H, Brück W, Nau R. Increased neuronal proliferation in human bacterial meningitis. Neurology. 2009;73:1026-1032.

43. Jiang W, Zhang Y, Xiao L, et al. Cannabinoids promote embryonic and adult hippocampus neurogenesis and produce anxiolytic- and antidepressant-like effects. J Clin Invest. 2005;115:3104-3116. 
44. Lee AL, Ogle WO, Sapolsky RM. Stress and depression: possible links to neuron death in the hippocampus. Bipolar Disord. 2002;4:117-128.

45. Sheline YI, Gado MH, Kraemer HC. Untreated depression and hippocampal volume loss. Am J Psychiatry. 2003;160:1516-1518.

46. Bjørnebekk A, Mathé AA, Brené S. The antidepressant effect of running is associated with increased hippocampal cell proliferation. Int $J$ Neuropsychopharmacol. 2005;8:357-368.

47. Lazarov O, Robinson J, Tang YP, et al. Environmental enrichment reduces Abeta levels and amyloid deposition in transgenic mice. Cell. 2005; 120:701-713.

48. Van Praag H, Kempermann G, Gage F. Running increases cell proliferation and neurogenesis in the adult mouse dentate gyrus. Nat Rev Neurosci. 1999;2:266-270.
49. Van Praag H, Shubert T, Zhao C, Gage F. Exercise enhances learning and hippocampal neurogenesis in aged mice. J Neurosci. 2005;25:8680-8685.

50. Aldskogius H. Microglia in neuroregeneration. Microsc Res Tech. 2001;54:40-46.

51. Bradbury EJ, McMahon SB. Spinal cord repair strategies: why do they work? Nat Rev Neurosci. 2006;7:644-653.

52. Huang H, Sharma HS. Neurorestoratology: one of the most promising new disciplines at the forefront of neuroscience and medicine. Journal of Neurorestoratology. 2013;1:37-41.

\section{Publish your work in this journal}

The Journal of Neurorestoratology is an international, peer-reviewed, open access online journal publishing original research and review articles on the subject of Neurorestoratology. To provide complete coverage of this revolutionary field the Journal of Neurorestoratology will report on relevant experimental research, technological advances, and

\section{Dovepress}

clinical achievements. The manuscript management system is completely online and includes a very quick and fair peer-review system, which is all easy to use. Visit http://www.dovepress.com/testimonials.php to read real quotes from published authors.

Submit your manuscript here: http://www.dovepress.com/journal-of-neurorestoratology-journal 